\title{
IMPACTS GÉNÉTIQUES DES REPEUPLEMENTS \\ EN TRUITES COMMUNES (SALMO TRUTTA L.) \\ SUR LES POPULATIONS EN PLACE : \\ CAS DU BASSIN DE L'ORB (HÉRAULT).
}

\author{
Dominique BEAUDOU (1), Ghislaine CATTANEO-BERREBI (2), P. BERREBI (2)*.
}

(1) Laboratoire d'Hydrobiologie Marine et Continentale, CNRS URA 1355, Université de Montpellier II, Place Eugène Bataillon, 34095 MONTPELLIER CEDEX 5 (France).

(2) Laboratoire Génome et Population, CNRS URA 1493, Université de Montpellier II, Place Eugène Bataillon, 34095 MONTPELLIER CEDEX 5 (France).

\section{RÉSUMÉ}

Depuis la fin du siècle dernier, pour pallier la réduction des populations de truites communes (Salmo trutta) liées aux activités humaines (pression de pêche, dégradation du milieu...), des pratiques de repeuplements se sont mises en place de façon plus ou moins généralisée. En région méditerranéenne, l'effet du repeuplement peut être suivi par électrophorèse des protéines, en particulier grâce à un locus diagnostique $\mathrm{LDH}-5^{\star}$; en effet, les souches de pisciculture, couramment utilisées, possèdent des fréquences élevées de l'allèle $L D H-5^{\star} 100$, récemment apparu (ère quaternaire), alors que les populations naturelles ont conservé l'allèle $L D H-5^{\star} 105$ méditerranéen ancestral. L'impact génétique des pratiques de repeuplement a été évalué sur trois populations du bassin de l'Orb, une sur le cours d'eau principal et les deux autres sur ses affluents. Tout le bassin de l'Orb est repeuplé depuis au moins une vingtaine d'années, mais avec des modalités différentes dans les zones échantillonnées. Malgré les déversements répétés, les populations naturelles échantillonnées possèdent de faibles fréquences de l'allèle 100 ( 3 à $14 \%$ ). L'impact génétique des déversements cumulés sur des décennies sur les populations de truites communes du bassin de l'Orb est donc faible.

Mots-clés : Salmo trutta fario, repeuplements, polymorphisme enzymatique, introgression.

\section{GENETIC IMPACTS OF TROUT (SALMO TRUTTA FARIO) STOCKING PRACTICES ON IN SITU POPULATIONS : CASE OF THE ORB BASIN (HERAULT).}

\begin{abstract}
The aim of this work is the evaluation of the genetic impact of the stocking practices, in the Orb basin, south of France. The effectiveness of the stocking policy, and its impact on the genetic pool of the natural populations, was followed in the Orb basin, where the number of trouts stocked each year between 1966 and 1989 was equivalent to that of the natural populations. Trouts collected by electro-fishing from this river and two of its tributaries, the Tes and the Mare, were characterized by starch gel electrophoresis at 25 loci. The three localities were stocked at least for twenty years, but the intensity of stocking and the stages used differ. The $\mathrm{LDH}-5^{*}$ locus, which codes for the eye-specific lactate deshydrogenase in brown trout, distinguishes between Atlantic and Mediterranean French populations, with two allelic forms respectively $L D H-5^{\star} 100$ and $L D H-5^{\star} 105$ (KRIEG and GUYOMARD, 1985). The hatchery reared trouts are Atlantic strains, that is why the $L D H-5^{*}$ represents a genetic detectable tag for the four hatchery strains, currently stocked in the Mediterranean populations
\end{abstract}

( $\left.{ }^{\star}\right)$ Auteur à qui les demandes de tirés à part doivent être adressées. 
of the Orb basin. The hatchery strains show high frequencies of $\mathrm{LDH}-5^{\star} 100(87.5$ to $100 \%)$. The three populations collected in the Orb basin show frequencies of $\mathrm{LDH}-5^{\star} 105$ between 86 to $97 \%$.

This high percentage of $L D H-5^{\star} 105$ shows the poor interbreeding between natural and hatchery-reared trouts. It may indicate that the stocked trouts do not reach the age of maturity.

Key-words : Salmo trutta fario, stocking practices, enzymatic polymorphism, introgression.

\section{INTRODUCTION}

La truite fario (Salmo trutta fario) est probablement l'espèce de poissons d'eau douce la plus manipulée par l'homme en Europe Occidentale. Depuis la fin du siècle dernier, grâce à la maîtrise de sa reproduction, l'homme a essayé de contrôler son abondance dans les cours d'eau par des repeuplements (THIBAULT, 1983). Ces pratiques, dans un premier temps sporadiques, sont rapidement devenues intensives, régulières et généralisées.

Un tel modèle biologique peut paraître totalement artificiel, mais plusieurs travaux ont montré, grâce à l'étude du polymorphisme enzymatique par électrophorèse des protéines, que malgré des déversements massifs depuis des décennies, le peuplement naturel a pu résister plus ou moins bien selon les endroits. Ces études sont notamment basées sur l'existence d'un marqueur génétique, la lactate déshydrogénase 5 de l'oeil. Que ce soit dans le bassin de l'Erne-Macnean en Irlande (TAGGART et FERGUSON, 1986), dans des cours d'eau méditerranéens français (BARBAT-LETERRIER et al.,1989) ou au Nord de l'Espagne (MORAN et al., 1991), des individus de pisciculture, porteurs de l'allèle moderne $L D H-5^{\star} 100$, sont déversés dans des populations possèdant l'allèle ancestral $L D H-5^{\star} 105$.

Nous nous sommes attachés à décrire l'impact génétique des repeuplements à l'échelle du bassin de l'Orb (Hérault), cours d'eau du sud de la France (passant à Béziers), où la situation est similaire. L'histoire biogéographique des populations de truites communes $a$, en effet, conduit à l'existence de ce marqueur génétique, en particulier en région méditerranéenne française. KRIEG et GUYOMARD (1985) ont montré que les populations françaises du versant atlantique, à l'exception de la Bretagne, possèdent l'allèle 100, alors que les populations méditerranéennes possèdent l'allèle 105 et que les populations de pisciculture, issues d'un faible nombre de souches, sont de caractéristique atlantique. Cette opposition reflète l'histoire biogéographique de l'espèce depuis les glaciations du quaternaire. Selon HAMILTON et al. (1989), l'allèle 105 est l'allèle ancestral puisqu'il existe chez 12 espèces de Salmonidés alors que l'allèle 100 est présent uniquement chez la truite commune. Le scénario proposé par ces auteurs est le suivant : l'allèle 105 était présent sur toute l'aire de répartition avant les perturbations glaciaires du quaternaire. Lors de celles-ci, les seules zones libres des glaces, constituant les zones refuges, étaient le sud de l'Europe, ainsi que le sud-ouest de l'Irlande et le sud de l'Angleterre. Les populations porteuses de la $L D H 5^{\star} 105$ se sont répandues avec le retrait des glaces. La forme $L D H-5^{\star} 100$ serait apparue dans la mer Baltique et les lles Britanniques. Elle a alors remplacé l'allèle 105 par introgression. La forme ancestrale s'est maintenue dans le bassin méditerranéen français (KRIEG et GUYOMARD, 1985), en Espagne (GARCIA-MARIN et al., 1991), en Grèce (KARAKOUSIS et TRIANTAPHYLLIDIS, 1990) et également dans des zones plus au nord ayant des barrières physiques à l'immigration (zones de hautes montagnes, ou isolées par des chutes infranchissables). La fréquence de l'allèle 105 est en effet plus forte dans les zones isolées d'Irlande, de Grande Bretagne et d'Islande (HAMILTON et al., 1989).

L'intérêt du bassin de l'Orb est de présenter sur le cours d'eau principal et sur les affluents des caractéristiques écologiques variées et des types de repeuplements divers, pratiqués depuis au moins une vingtaine d'années (*) (BEAUDOU et al., 1994). Ce cours d'eau prend sa source dans les Causses de Romiguières (Aveyron), à $825 \mathrm{~m}$ d'altitude, et

(") Les repeuplements sont probablement pratiqués depuis plus longtemps. Cependant, les premiers bilans annuels ne sont disponibles qu'à partir de 1966 (bilans d'alevinage de la Fédération des Associations pour la Pêche et la Protection des Milieux Aquatiques de ('Hérault). 
se jette après un parcours de $136 \mathrm{~km}$ dans la Méditerranée. Trois populations de truites communes ont été analysées, une sur le cours d'eau principal et deux sur des affluents, choisis pour leurs caractéristiques écologiques différentes, la Tes et la Mare. La Tes, avec son principal affluent le Tirounan, est un petit cours d'eau salmonicole $(9,5 \mathrm{~km})$ situé à l'amont du bassin. Elle présente une pente à peu près constante (19\%) avec des décrochements créés par des tables calcaires. La Mare, est un cours d'eau plus important (36 km de long), circulant essentiellement sur roches cristallines. Son principal affluent, le Bouissou, apporte une part importante du débit grâce à son alimentation par une source karstique. La portion salmonicole étudiée correspond à la partie amont, avec une pente moyenne de $18 \%$.

\section{MATÉRIEL ET MÉTHODES}

Les échantillons des populations en place ont été collectés en individualisant les différentes sous-unités de gestion, Tes, Mare et Orb (fig. 1 et tab. I). Alors que la Mare et l'Orb reçoivent chaque année des alevinages, sur la Tes les repeuplements ont été suspendus depuis janvier 1988. Les échantillons ont été prélevés lors de pêches électriques durant l'été 1991. Pour la Tes et la Mare, ils représentent le cumul d'échantillons réalisés sur trois secteurs de ces cours d'eau (fig. 1 et tab.l) et ont été réalisés de façon à obtenir des échantillons suffisamment importants de juvéniles de l'année $(0+)$ et de poissons plus âgés $(\geq 1+)$. Les fréquences alléliques dans la population sont obtenues en additionnant les fréquences alléliques des $0+$ pondérées par la proportion de juvéniles dans la population totale et les fréquences alléliques des poissons plus âgés pondérées par leur proportion dans la population totale. La Mare et la Tes, au mois de juillet, comptent respectivement $47,5 \%$ et $83,3 \%$ de juvéniles. Pour la Tes, le sous-échantillon de poissons plus âgés correspond à un prélèvement du mois d'avril.

Tableau I: Caractéristiques des sites d'échantillonnage du bassin de l'Orb et composition des échantillons naturels.

Table I : Characteristics of the Orb basin sampling localities and natural samples composition.

\begin{tabular}{|c|c|c|c|c|c|c|c|}
\hline \multirow{2}{*}{$\begin{array}{l}\text { Echantillon } \\
\text { Site }\end{array}$} & \multicolumn{3}{|c|}{ Tes et Tirounan } & \multirow{2}{*}{$\frac{\text { Orb }}{D}$} & \multicolumn{3}{|c|}{ Mare } \\
\hline & A & $B$ & $\mathrm{C}$ & & $E$ & $F$ & G \\
\hline $\begin{array}{l}\text { Altitude }(\mathrm{m}) \\
\text { Distance aux sources }(\mathrm{km}) \\
\text { Pente }(\%) \\
\text { Largeur }(\mathrm{m})\end{array}$ & $\begin{array}{c}530 \\
3 \\
16 \\
3\end{array}$ & $\begin{array}{c}502 \\
2,5 \\
15 \\
5,5\end{array}$ & $\begin{array}{c}459 \\
16 \\
16 \\
5,5\end{array}$ & $\begin{array}{c}354 \\
19 \\
11 \\
16\end{array}$ & $\begin{array}{l}370 \\
5,5 \\
20 \\
4,3\end{array}$ & $\begin{array}{c}320 \\
9,5 \\
8 \\
8,3\end{array}$ & $\begin{array}{c}300 \\
11 \\
11,5 \\
7,2\end{array}$ \\
\hline $\begin{array}{l}\text { Densité de truites } / 100 \mathrm{~m}^{2} \\
\text { Biomasse de truites } / 100 \mathrm{~m}^{2} \\
\text { Biomasse totale/100 } \mathrm{m}^{2}\end{array}$ & $\begin{array}{c}100 \\
1,3 \mathrm{~kg} \\
1,3 \mathrm{~kg}\end{array}$ & $\begin{array}{c}69 \\
1,5 \mathrm{~kg} \\
1,5 \mathrm{~kg}\end{array}$ & $\begin{array}{c}77 \\
1,6 \mathrm{~kg} \\
1,8 \mathrm{~kg}\end{array}$ & $\begin{array}{c}20 \\
0,8 \mathrm{~kg} \\
1,4 \mathrm{~kg}\end{array}$ & $\begin{array}{c}8 \\
0,4 \mathrm{~kg} \\
0,6 \mathrm{~kg}\end{array}$ & $\begin{array}{c}22 \\
1,3 \mathrm{~kg} \\
2,9 \mathrm{~kg}\end{array}$ & $\begin{array}{c}6 \\
0,4 \mathrm{~kg} \\
6,2 \mathrm{~kg}\end{array}$ \\
\hline $\begin{array}{cc}\text { Prélèvements } & \begin{array}{c}\text { Date } \\
\text { Cohortes } \\
\text { Nombre }\end{array}\end{array}$ & \multicolumn{3}{|c|}{$\begin{array}{ccc}18 / 4 / 91 & 16 / 7 / 91 \\
0+ & - & \geq 1+ \\
18 & - & 31\end{array}$} & $\begin{array}{c}19 / 6 / 91 \\
\geq 1+ \\
15\end{array}$ & & $\begin{array}{l}11 / 7 / 91 \\
+\quad-\quad \geq \\
3 \quad-\quad 17\end{array}$ & $7^{1+}$ \\
\hline
\end{tabular}

Les quatre souches de pisciculture le plus couramment utilisées ces dernières années pour le repeuplement du bassin de l'Orb ont également été analysées. II s'agit des souches des piscicultures de Brassac (Tarn), de la Canourgue (Lozère), de la Mouline (Aveyron) et du Tyrol italien. Les échantillons de la pisciculture de la Canourgue et de la Mouline ont été prélevés sur place. Les truites originaires de la pisciculture de Brassac et du Tyrol Italien ont été fournies par la pisciculture fédérale de Fontcaude (Hérault), où s'effectue le grossissement des individus destinés aux repeuplements. 


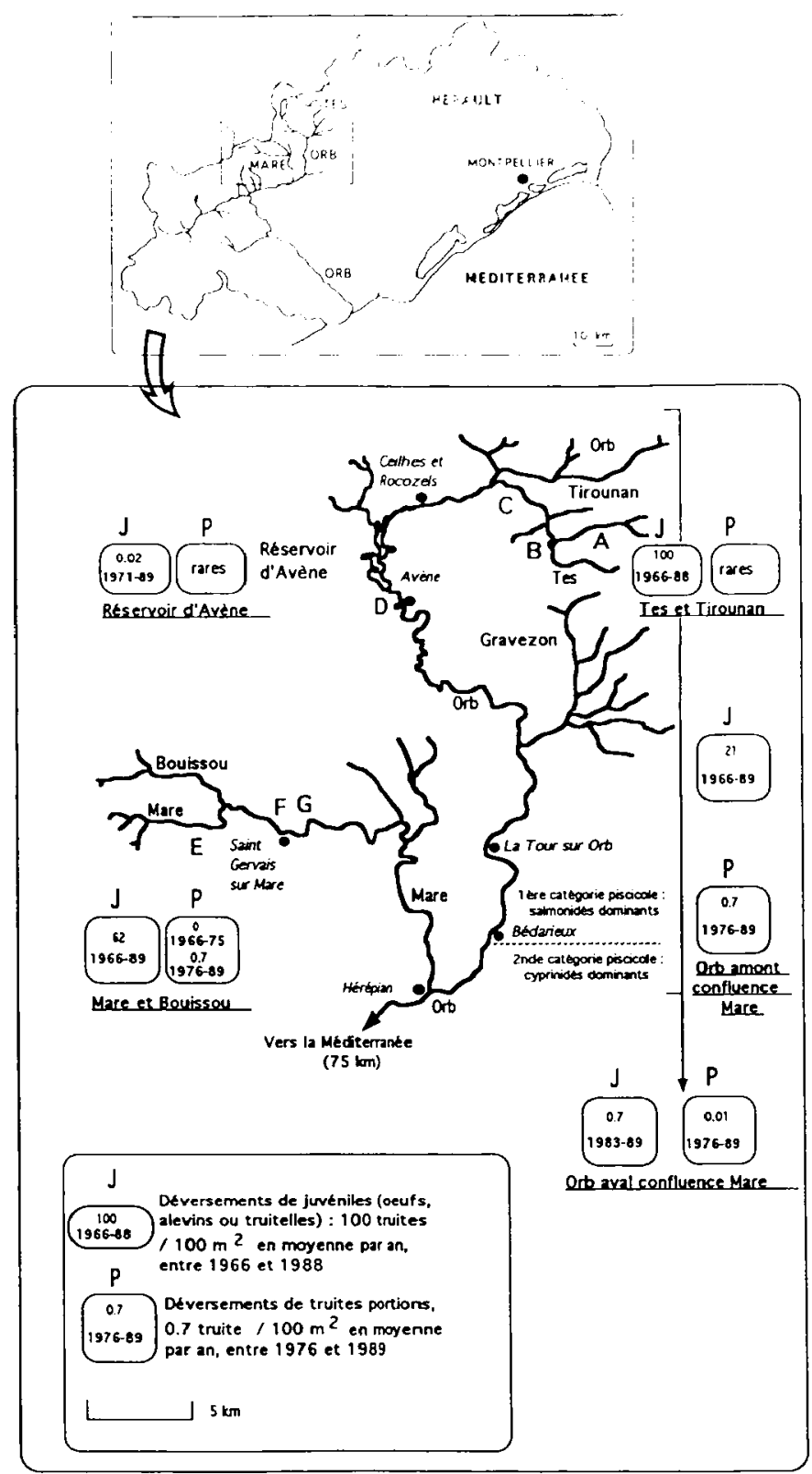

Figure 1 : Localisation des sites d'échantillonnage et repeuplements moyens annuels en truites communes dans le bassin de l'Orb entre 1966 et 1989. J représente les déversements moyens de juvéniles (oeufs, alevins et truitelles de l'année) et $P$ correspond aux lâchers d'individus portions, ramenés à $100 \mathrm{~m}^{2}$ de cours d'eau. Par exemple, la Mare reçoit en moyenne par an 62 juvéniles/100 $\mathrm{m}^{2}$ de surface de 1ère catégorie piscicole (de 1966 à 1989), et 0,7 truite "portion» pour $100 \mathrm{~m}^{2}$ de cours total (de 1976 à 1989), alors que les densités naturelles évoluent entre 6 et 23 individus pour $100 \mathrm{~m}^{2}$.

Figure 1 : Sampling localities and stocking of the Orb basin with brown trout between 1966 and 1989. J represents the annual mean of juveniles stockings (eggs, fry and yearlings) for $100 \mathrm{~m}^{2}, P$ represents the catchable trouts stocked annually in mean for $100 \mathrm{~m}^{2}$. For example, in the Mare river, in mean, 62 juveniles/100 $\mathrm{m}^{2}$ of first class of the fish culture were stocked each year between 1966 and 1989 , and 0.7 catchable trout $/ 100 \mathrm{~m}^{2}$ of total surface was stocked between 1976 and 1989 at the same time. 
Les truites sont transportées vivantes au laboratoire et congelées à $-20{ }^{\circ} \mathrm{C}$. Des échantillons d'oeil, de muscle et de foie sont prélevés et broyés avec un volume équivalent de tampon Tris-HCL-EDTA-NADP $(\mathrm{pH}=8)$. Après centrifugation, le surnageant est prélevé et conservé à $-75^{\circ} \mathrm{C}$.

Les électrophorèses se font sur gels d'amidon horizontaux, selon la technique décrite par PASTEUR et al. (1987), avec les tampons et les colorations de KRIEG (1984).

Quatorze systèmes enzymatiques ont été étudiés : aspartate aminotransférase SAAT (E.C. 2.6.31.1), alcool déhydrogénase ADH (E.C. 1.1.1.1), créatine kinase CK (E.C. 2.7.3.2), fructose-biphosphatase FBP (E.C. 3.1.3.11), fumarate hydratase FH (E.C. 4.2.1.2), glucose-6phosphate isomérase GPI (E.C. 5.3.1.9), glycérol-3-phosphate déshydrogénase G3PDH (E.C. 1.1.1.8), guanine déaminase GDA (E.C. 3.5.4.3), isocitrate déhydrogénase $\left(\mathrm{NADP}^{+}\right)$IDHP (E.C. 14.1.1.42), L-lactate déshydrogénase LDH (E.C. 1.1.1.27), malate déhydrogénase SMDH (E.C. 1.1.1.37), mannose-6-phosphate isomérase MPI (E.C. 5.3.1.8), phosphoglucomutase PGM (E.C. 5.4.2.2), phosphogluconate déshydrogénase PGDH (E.C. 1.1.1.44), soit 28 locus. Seize locus sont polymorphes avec deux allèles chacun. Pour le locus $L D H-5^{*}$, les deux allèles observés (100 et 105 ) sont définis par comparaisons directes sur gels avec des individus étudiés par R. GUYOMARD. Pour les allèles à forte fréquence (ceux des locus sAAT-1*, SAAT-4*,CK-1*, G3PDH-2*, FDP-1*, $\left.s M D H-2^{*}, s M D H-3^{\star}, M P I-2^{\star}\right)$, la dénomination des différents allèles est faite par référence à deux souches de pisciculture étudiées par KRIEG (1984) (souche du Tyrol italien de la pisciculture Magnet) et BARBAT-LETERRIER et al. (1989) (souche de la Canourgue). Bien que l'élevage puisse induire une perte de polymorphisme (VUORINEN, 1984), il est peu probable que des allèles à forte fréquence puissent disparaître rapidement. Pour les allèles à faible fréquence (IDHP-3*201 et $P G D H-2 * 86)$, en l'absence de comparaison directe à des échantillons déjà décrits, la dénomination a été réalisée en rajoutant 1 au numéro des allèles déjà observés dans les populations françaises (KRIEG, 1984). De même, pour la fumarate hydratase, l'allèle rapide $\left(^{*}\right)$ est noté 135 , parce que nous ne pouvons différencier les allèles 130 et 140 observés par KRIEG (1984), bien que ces deux allèles soient présents dans la Tes et l'Orb (comparaison directe avec individus analysés par R. GUYOMARD). Les deux locus observés sont des locus dupliqués partageant les mêmes allèles. Comme les différents hétérozygotes ne sont pas différenciés $\mathrm{FH}-1 * 100 / 100$ et $\mathrm{FH}-2 * 100 / 135$ ou $\mathrm{FH}-1 * 100 / 135$ et $\mathrm{FH}-2^{*} 100 / 100, \mathrm{FH}-1^{*} 100 / 100$ et $\mathrm{FH}-2^{*} 135 / 135$ ou $\mathrm{FH}-1^{*} 135 / 135$ et $\mathrm{FH}-2 * 100 / 100$, $\mathrm{FH}-1^{\star} 100 / 135$ et $\mathrm{FH}-2^{*} 135 / 135$ ou $\mathrm{FH}-1^{*} 135 / 135$ et $\mathrm{FH}-2^{\star} 100 / 135$, seules les fréquences phénotypiques (homozygote lent, homozygote rapide et hétérozygote noté $\mathrm{FH}-1,2 \mathrm{H}$ ) sont calculées. Pour $C K-1^{*}$, nous ne pouvons différencier les hétérozygotes (CK-1*100/125) des homozygotes lents $(C K-1 * 100 / 100)$. Les fréquences ont donc été calculées à partir de la racine carrée de la fréquence de l'homozygote discernable (CK-1*125/125), en supposant que la population échantillonnée était en panmixie, obéissant aux proportions génotypiques de la loi de Hardy-Weinberg.

\section{RÉSULTATS}

Les fréquences alléliques des locus polymorphes sont présentées dans le tableau 11.

Le locus $\mathrm{LDH}-5^{\star}$ est le plus informatif, puisque défini comme diagnostique par GUYOMARD (1989) (un locus diagnostique ne présente aucun allèle commun entre les taxons à différencier et suffit à lui seul à rattacher un individu à l'un ou l'autre de ces taxons). Conformément à ses observations, les souches de pisciculture étudiées possèdent essentiellement l'allèle atlantique (fig. 2). Elles montrent cependant de faibles fréquences de l'allèle méditerranéen, $L D H-5^{*} 105(0$ à $3 \%)$, à l'exception de l'échantillon de la pisciculture de la Mouline (12,5\%). II s'agit probablement de la marque de l'utilisation plus ou moins épisodique de géniteurs locaux. Inversement, les échantillons naturels ont conservé de fortes fréquences de l'allèle ancestral ( 86 à $97 \%$ ), indicatrices de la disparition des individus de repeuplement. Les fréquences de l'allèle $\mathrm{LDH}-5^{*} 105$ observées pour les échantillons de juvéniles $(0+)$ et pour les autres classes d'âge $(\geq 1+)$ ne diffèrent pas significativement au seuil de $5 \%$ (test exact de Fisher (SCHERRER, 1984))

(*) L'allèle rapide correspond à l'allèle le plus mobile, l'allèle lent à l'allèle le moins mobile dans les conditions de migration utilisées. 
Tableau II : Fréquences alléliques des locus polymorphes pour les souches de pisciculture et les populations naturelles $(n=$ nombre d'individus analysés pour chaque locus).

$\Sigma$ : pour les échantillons de la Tes et de la Mare, la fréquence dans la population $=$ [fréquence allélique chez les juvéniles $(0+)$ x proportion de juvéniles dans la population totale] + [fréquence allélique chez les autres classes d'âge $(\geq 1+) \times$ proportion dans la population totale].

Table II : Allelic frequencies of the polymorphic loci for the four hatchery strains (Brassac, Canourgue, Italian Tyrol, Mouline) and the three populations of the Orb basin (Orb at la Prade, Tes and Mare) ( $n=$ number of individuals analysed for each locus).

$\Sigma:$ for the Tes and the Mare samples, the population allelic frequency is corrected by the ratio young/adult. Hatchery strains show high frequencies of $L D H 5^{\star} 100(87.5$ to $100 \%)$. In spite of stocking practices, the river populations have conserved the ancestral allele, $L D H 5{ }^{\star} 105$ (86 to $97 \%$ ).

\begin{tabular}{|c|c|c|c|c|c|c|c|c|c|c|c|c|}
\hline \multirow[b]{4}{*}{ Locus } & \multicolumn{8}{|c|}{ Populations naturelies } & \multicolumn{4}{|c|}{ Souches de pisciculture } \\
\hline & & \multicolumn{4}{|c|}{ TES ET TIROUNAN $(A+B+C)$ ORB (D) } & \multicolumn{3}{|c|}{$\operatorname{MARE}(E+F+G)$} & \multirow{3}{*}{$\frac{\text { Brassac }}{1811 / 91}$} & \multicolumn{2}{|c|}{ Canourgue Tyrol Italien } & \multirow{3}{*}{$\begin{array}{l}\text { Mouline } \\
05 / 06 / 90\end{array}$} \\
\hline & & \multicolumn{3}{|c|}{$16 / 07 / 91 \quad 18 / 04 / 91$} & \multicolumn{2}{|l|}{$19 / 06 / 91$} & \multicolumn{2}{|c|}{$19107 / 91$} & & \multirow[t]{2}{*}{$31 / 01 / 90$} & \multirow{2}{*}{$09 / 11 / 90$} & \\
\hline & & $\mathrm{L}^{0+}$ & $\geq 1+$ & $\Sigma$ & $>0+$ & $0+$ & $\geq 1+$ & $\Sigma$ & & & & \\
\hline \multirow[t]{3}{*}{ SAAT-1" } & 100 & 1.00 & 0.98 & 100 & 1.00 & 1.00 & 1.00 & 1.00 & 0.93 & 087 & 0.955 & 080 \\
\hline & 130 & & 0.02 & & & & & & 0.07 & 0.13 & 0.045 & 0.20 \\
\hline & $n$ & 17 & 31 & 48 & 15 & 11 & 14 & 25 & 30 & 15 & 11 & 28 \\
\hline \multirow[t]{4}{*}{ SAAT $-4^{\circ}$} & 65 & & 0.02 & & & & 0.03 & 0.02 & 0.18 & 0.37 & 0.05 & 0.16 \\
\hline & 100 & 1.00 & 0.98 & 1.00 & 1.00 & 1.00 & 0.97 & 0.98 & 0.81 & 0.63 & 0.95 & 0.80 \\
\hline & 110 & & & & & & & & 0.01 & & & 0.04 \\
\hline & $\mathbf{n}$ & 13 & 31 & 44 & 15 & 13 & 17 & 30 & 31 & 15 & 10 & 28 \\
\hline \multirow[t]{3}{*}{$C K-1$} & 100 & 1.00 & 1.00 & 100 & 1.00 & 1.00 & 1.00 & 1.00 & 0.73 & 0.63 & 100 & 0.62 \\
\hline & 125 & & & & & & & & 0.27 & 0.37 & & 0.38 \\
\hline & $n$ & 16 & 31 & 47 & 15 & 13 & 17 & 30 & 28 & 15 & 9 & 28 \\
\hline$F B P-1^{*}$ & 100 & & 0.06 & 0.01 & 0.10 & 0.06 & & 0.03 & 0.905 & 0.87 & 0.86 & 0.68 \\
\hline & 150 & 1.00 & 0.94 & 0.99 & 0.90 & 0.94 & 1.00 & 0.97 & 0.095 & 0.13 & 0.14 & 0.32 \\
\hline & $n$ & 12 & 26 & 38 & 15 & 9 & 17 & 26 & 21 & 15 & 7 & 25 \\
\hline $\mathrm{FH}-1,2^{*}$ & 135 & & & & 2 & & & & 6 & & 1 & 12 \\
\hline & hetéro. & & 3 & 3 & & & 1 & 1 & 8 & 6 & & 11 \\
\hline & 100 & 17 & 25 & 42 & 13 & 12 & 16 & 28 & 16 & 9 & 11 & 3 \\
\hline & $n$ & 17 & 28 & 45 & 15 & 12 & 17 & 29 & 30 & 15 & 12 & 26 \\
\hline GDA* & 50 & & 0.17 & & & & & & & 0.67 & & 0.63 \\
\hline & 100 & 1.00 & 0.83 & & 1.00 & 1.00 & 1.00 & 1.00 & 1.00 & 0.33 & 1.00 & 0.37 \\
\hline & $n$ & 18 & 29 & 47 & 15 & 13 & 17 & 30 & 30 & 15 & 12 & 27 \\
\hline G3POH-2* & 50 & & 0.02 & & 0.04 & 0.27 & 0.35 & 0.31 & 0.16 & 0.13 & 0.21 & 0.27 \\
\hline & 100 & 1.00 & 0.98 & 1.00 & 0.96 & 0.73 & 0.65 & 0.69 & 0.84 & 0.87 & 0.79 & 0.73 \\
\hline & $n$ & 18 & 31 & 49 & 14 & 13 & 17 & 30 & 31 & 15 & 12 & 28 \\
\hline GPI.2* & 100 & 0.89 & 0.97 & 0.90 & 1.00 & 0.89 & 0.94 & 0.92 & 1.00 & 1.00 & 1.00 & 0.8 \\
\hline & 200 & 0.11 & 0.03 & 0.10 & & 0.11 & 0.06 & 0.08 & & & & 0.2 \\
\hline & $n$ & 18 & 29 & 47 & 15 & 13 & 17 & 30 & 31 & 15 & 12 & 23 \\
\hline IDHP-1* & 90 & 0.03 & & 0.02 & & & & & & & & 0.02 \\
\hline & 100 & 0.97 & 1.00 & 0.98 & 1.00 & 1.00 & 1.00 & 1.00 & 1.00 & 1.00 & 1.00 & 0.98 \\
\hline & $n$ & 18 & 31 & 49 & 15 & 13 & 17 & 30 & 30 & 15 & 12 & 27 \\
\hline IDHP-3* & 100 & 1.00 & 1.00 & 1.00 & 1.00 & 1.00 & 1.00 & 1.00 & 1.00 & 1.00 & 1.00 & $0 . \pi 7$ \\
\hline & 201 & & & & & & & & & & & 0.23 \\
\hline & $n$ & 18 & 28 & 46 & 15 & 13 & 17 & 30 & 30 & 15 & 12 & 27 \\
\hline $\mathrm{LOH}-5^{\circ}$ & 100 & 0.16 & 0.06 & 0.14 & 0.03 & 0.17 & 0.12 & 0.14 & 0.98 & 0.97 & 1.00 & 0.875 \\
\hline & 105 & 0.84 & 0.94 & 0.86 & 0.97 & 0.83 & 0.68 & 0.86 & 0.02 & 0.03 & & 0.125 \\
\hline & $n$ & 16 & 31 & 47 & 15 & 12 & 17 & 29 & 31 & 15 & 12 & 28 \\
\hline SMDH-2* & 100 & 0.71 & 0.92 & 0.75 & 1.00 & 0.92 & 0.94 & 0.93 & 0.855 & $0 \pi$ & 0.92 & 0.55 \\
\hline & 200 & 0.29 & 0.08 & 0.25 & & 0.08 & 0.06 & 0.07 & 0.145 & 0.23 & 0.08 & 0.45 \\
\hline & $n$ & 17 & 31 & 48 & 15 & 13 & 17 & 30 & 31 & 15 & 12 & 28 \\
\hline SMDH-3* & 75 & 0.06 & & 0.05 & & 0.08 & 0.19 & 0.14 & 0.15 & 0.33 & 0.32 & 0.22 \\
\hline & 100 & 0.94 & 1.00 & 0.95 & 1.00 & 0.92 & 0.81 & 0.86 & 0.85 & 0.67 & 0.68 & 0.78 \\
\hline & $\mathrm{n}$ & 18 & 31 & 49 & 15 & 13 & 16 & 29 & 30 & 15 & 11 & 27 \\
\hline MPI-2* & 95 & & & & & & & & & & & 0.15 \\
\hline & 100 & 0.59 & 0.61 & 0.59 & 0.82 & 0.875 & 0.97 & 0.92 & 0.32 & 0.57 & 0.54 & 0.44 \\
\hline & 105 & 0.41 & 0.39 & 0.41 & 0.18 & 0.125 & 0.03 & 0.08 & 0.68 & 0.43 & 0.46 & 0.41 \\
\hline & $n$ & 17 & 31 & 48 & 14 & 12 & 17 & 29 & 31 & 14 & 12 & 27 \\
\hline PGDH-2* & 86 & & & & & & & & & & & 0.09 \\
\hline & 100 & 1.00 & 1.00 & 1.00 & 1.00 & 1.00 & 1.00 & 1.00 & 1.00 & 1.00 & 1.00 & 0.91 \\
\hline & $n$ & 18 & 31 & 49 & 15 & 13 & 17 & 30 & 31 & 15 & 10 & 27 \\
\hline No total indir & dus & 18 & 31 & 49 & 15 & 13 & 17 & 30 & 31 & 15 & 12 & 28 \\
\hline
\end{tabular}




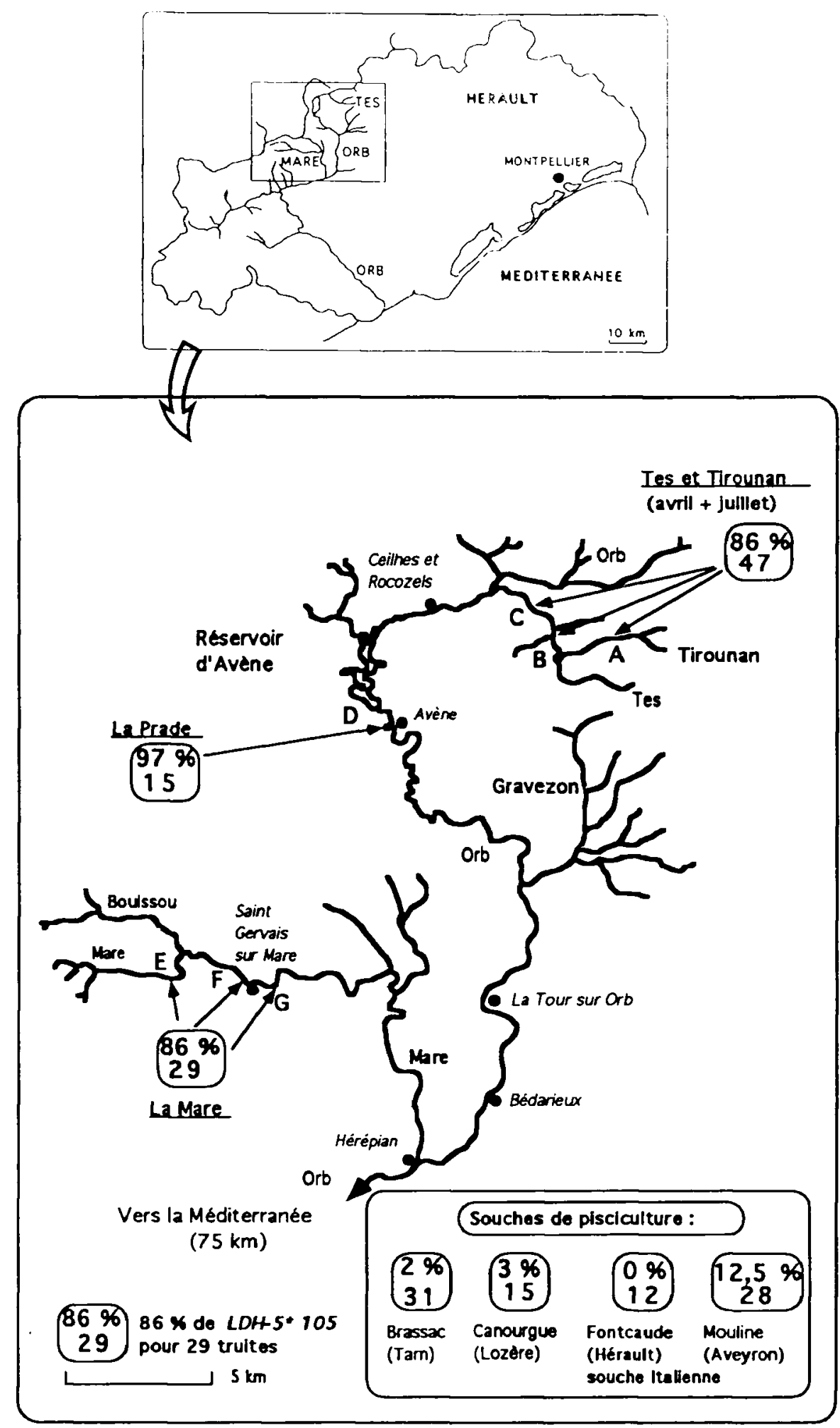

Figure 2 : Fréquences de l'allèle $L D H-5^{*} 105$ dans trois populations du bassin de l'Orb et quatre souches de pisciculture couramment utilisées pour le repeuplement.

Figure 2 : Frequencies of $L D H-5^{\star} 105$ allele in three Orb basin populations and four hatchery strains currently used for stocking. 
Le locus $L D H-5^{\star}$ n'est pas le seul système informatif. Un autre locus, FBP-1*, présente de fortes différences de fréquences entre les souches de pisciculture, qui possèdent entre 68 et $90 \%$ de l'allèle 100 et les populations sauvages, qui n'en présentent que 1 à $10 \%$. Ces différences de fréquences élevées confirment le faible maintien des individus de pisciculture dans le milieu naturel. Cependant, ce locus n'est pas diagnostique à l'inverse de ce que l'on observe dans le lac d'Arratille (Hautes-Pyrénées) (BERREBI et BEAUDOU, 1992), où la souche sauvage est fixée pour l'allèle 150 et la souche déversée pour l'allèle 100 .

La souche de la Mouline est plus polymorphe que les autres. Elle possède notamment les allèles $I D P H-3^{\star} 201, M P I-2^{*} 95$ et $P G D H-2^{\star} 86$, absents des autres souches. Bien que ces allèles soient présents à des fréquences assez faibles (respectivement $23 \%, 15 \%$ et $9 \%$ ), leur absence des échantillons naturels est un indicateur de la disparition des individus de repeuplements. De la même manière, l'allèle sAAT-4*65, présent entre 5 et $37 \%$ dans les souches de pisciculture, est très peu fréquent dans les échantillons naturels ( 0 à $2 \%$ ).

\section{DISCUSSION}

La seule observation du tableau des fréquences alléliques permet d'observer que les déversements importants des dernières décennies n'ont que très partiellement introgressé la forme locale sauvage de l'Orb. Les populations naturelles présentent, en effet, de fortes fréquences d'un allèle endémique de la région méditerranéenne ( 86 à $97 \%$ de $L D H-5^{\star} 105$ ). Or, ces déversements représentent chaque année au moins l'effectif de la population en place (toutes classes d'âge confondues) (tab. II, fig. 1 et fig. 2 ; BEAUDOU et al., 1994). La présence d'hétérozygotes $\mathrm{LDH}-5^{\star}$ 100/105 confirme l'interfécondité des deux origines.

Les fréquences de $L D H-5^{*} 105$ observées dans les trois échantillons naturels ne diffèrent pas significativement (test exact de Fisher à $5 \%$ ), malgré la variété des pratiques piscicoles et des milieux récepteurs. En effet, les repeuplements sont suspendus depuis début 1988 sur la Tes, cours d'eau salmonicole de tête de bassin, présentant un bon potentiel de reproduction naturel. La Mare est une rivière perturbée (débit d'étiage faible, aggravé par des prélèvements d'eau), plus cloisonnée (nombreux seuils permettant l'irrigation) avec des populations salmonicoles moins importantes en effectifs.

Les différentes études de suivi génétique des repeuplements en truites communes aboutissent à des résultats contrastés. MORAN et al. (1991) ont analysé des échantillons de truites de trois rivières du Nord de l'Espagne, avec des zones repeuplées et des zones sans déversement, à l'aide du marqueur $L D H-5^{\star}$. Sur les sites repeuplés, ils observent de faibles pourcentages d'individus de pisciculture $(0 \%, 5 \%$ et $7 \%)$, sauf pour une station où la reproduction naturelle est impossible et où le peuplement est composé uniquement d'individus de pisciculture. Dans ces échantillons, l'absence d'hétérozygotes $L D H-5^{\star} 100 / 105$ laisse penser que les juvéniles déversés n'atteignent pas la maturité. TAGGART et FERGUSON (1986) observent des introgressions variant de 19 à $91 \%$ sur 12 sites repeuplés du bassin de l'Erne-Macnean, au nord-ouest de l'Irlande. Ces taux d'introgression par la souche de pisciculture sont calculés en tenant compte du faible pourcentage de marqueur $L D H-5^{\star} 105$ présent dans cette souche ( $9 \%$ en moyenne) et en prenant comme référence des échantillons de zones non repeuplées. BARBAT-LETERRIER et al. (1989) observent des taux d'introgression multi-locus moyens variant de 0 à $40 \%$ sur trois cours d'eau méditerranéen, affluents du Var (Alpes-de-Haute-Provence), de la Cèze (Gard), et du Tech (Pyrénées-Orientales). Comme précédemment, ces taux d'introgression sont calculés en tenant compte de la fréquence de marqueur sauvage dans la souche de pisciculture et en se référant aux fréquences alléliques d'échantillons issus de zones non repeuplées. GUYOMARD et KRIEG (1986) citent des taux d'introgression moyens de 0 à $50 \%$ pour 4 localités d'une rivière Corse, la Rizzanèse. II est donc probable que des cas forts différents peuvent être rencontrés et que les caractéristiques historiques, humaines et écologiques des différents cours d'eau expliquent cette diversité.

Dans ce travail sur le bassin de l'Orb, les taux d'introgression ne sont pas standardisés par rapport à des zones indemnes de tout repeuplement. La détermination de tels secteurs se heurte à deux difficultés : l'absence d'enregistrement de certains déversements et le manque de localisation précise des lieux de lâchers. D'autre part, la 
présence de l'allèle 105 en pisciculture ne peut servir à pondérer les estimations car, contrairement aux travaux précédemment cités, plusieurs souches de pisciculture sont utilisées simultanément pour repeupler les mêmes zones. Bien qu'une souche soit généralement utilisée préférentiellement pour un stade de déversement, il est difficile d'évaluer précisément le pourcentage de marqueur $\mathrm{LDH}-5^{*} 105$ introduit par les déversements, ce pourcentage variant suivant les souches.

La technique employée dans cette étude ne rend pas compte des déversements de truites "portions" effectués avant et pendant la période de pêche. Ces poissons "panier" sont repêchés rapidement (CRESSWELL et WILLIAMS, 1982) et ils n'influent donc pas sur le pool génétique de la population en place. L'étude génétique des populations naturelles ne permet pas d'évaluer véritablement l'efficacité des repeuplements en terme de captures par les pêcheurs, bien qu'elle mette en évidence une disparition massive et rapide de la plupart des individus déversés. En effet, pour évaluer précisément le nombre de prises dues aux repeuplements, les caractérisations électrophorétiques doivent s'appliquer aux individus capturés. De cette manière, GARCIA DE LEÁNIZ et al. (1989) ont par exemple évalué les contributions respectives des oeufs sauvages et des oeufs de repeuplement aux prises de saumons (Salmo salar L.), sur deux rivières espagnoles. Les populations sauvages possèdent de fortes fréquences de l'allèle $M E-2 * 100$ (85 à $100 \%$ ), alors que la souche de pisciculture utilisée, originaire d'Ecosse, présente une fréquence plus faible de cet allèle $(33,7 \%)$. La contribution des oeufs de repeuplement aux captures par les pêcheurs est très inférieure à celle des oeufs sauvages sur les deux cours d'eau étudiés (respectivement 0,9 captures et 0 pour 10000 oeufs de repeuplement contre 4,1 et 4,9 captures pour 10000 oeufs sauvages).

\section{CONCLUSION}

La présente étude sur les populations de Salmo trutta fario du bassin de l'Orb a montré que la génétique des populations peut apporter des réponses nuancées et en profondeur (effet cumulé de tous les déversements effectués) aux questions d'efficacité des repeuplements. Sur ce bassin, la souche sauvage ancestrale s'est maintenue, bien qu'elle ait été partiellement introgressée par les souches de pisciculture déversées. Cependant, compte tenu de l'ampleur des déversements, nous pouvons conclure à une faible efficacité des repeuplements. essentielles :

La technique d'électrophorèse des protéines permet de répondre à des questions

- existe-t-il une souche sauvage à préserver et est-elle vraiment différente des souches voisines?

- les repeuplements en truites de pisciculture sont-ils efficaces et ont-ils modifié à la longue les caractéristiques des truites sauvages? Quel est l'avenir des souches sauvages locales?

II faut cependant noter que cette technique a montré son efficacité dans le cas de repeuplements en truites de pisciculture (d'origine atlantique) en région méditerranéenne. Pour effectuer les mêmes analyses en région atlantique, d'autres marqueurs, non enzymatiques, doivent être mis au point, par analyse directe de l'ADN mitochondrial ou nucléaire. De telles études sont actuellement en cours.

Quand, dans une région donnée, l'ensemble de ces réponses a été établi, le gestionnaire a toutes les cartes en main pour agir suivant l'objectif qu'il s'est fixé : entretenir artificiellement un peuplement en truites de pisciculture ou préserver une souche locale.

\section{REMERCIEMENTS}

Cette étude a été réalisée grâce à des financements du Conseil Supérieur de la Pêche et de la Fédération des Associations Agréées de Pêche et de Protection des Milieux Aquatiques de l'Hérault. Nos remerciements vont à la $8^{e}$ Délégation Régionale du Conseil Supérieur de la Pêche, à la garderie de la Fédération des APPMA de l'Hérault et aux bénévoles de l'APPMA de Bédarieux qui ont permis la réalisation des échantillonnages, et également à $G$. LASSERRE, $B$. DOD et $P$. BORSA pour la relecture critique du manuscrit. 


\section{BIBLIOGRAPHIE}

BARBAT-LETERRIER A., GUYOMARD R., KRIEG F., 1989. Introgression between introduced domesticated strains and mediterranean native populations of brown trout (Salmo trutta L.). Aquat. Living. Resour., 2, 215-223.

BEAUDOU D., DE ROBERT A., BERREBI P., 1994. Pratiques de repeuplements en région méditerranéenne française : cas du bassin de l'Orb (Hérault). Bull. Fr. Pêche et Piscic., 332.

BERREBI P., BEAUDOU D., 1992. La génétique des populations appliquée à la truite fario : les cas de l'Orb (Hérault, France) et des Pyrénées (France et Andorre). Actes du colloque "Truite commune pyrénéenne : génétique et repeuplement", Oronoz (Espagne), 26 novembre 1991, 5-19.

CRESSWELL R.C., WILLIAMS R., 1982. Post-stocking movements and recapture of hatchery trout released into flowing waters: effect of time and method of stocking. Fish. Mgmt, 13 (3), 97-103.

GARCIA DE LEÁNIZ C., VERSPOOR E., HAWKINS A.D., 1989. Genetic determination of the contribution of stocked and wild Atlantic salmon, Salmo salar L., to the angling fisheries in two Spanish rivers. J. Fish Biol., 3 (suppl. A), 261-270.

GARCIA-MARIN J.L., JORDE P.E., RYMAN N., UTTER F., PLA C., 1991. Management implications of genetic differentiation between native and hatchery populations of brown trout (Salmo trutta) in Spain. Aquaculture, 95, 235-249.

GUYOMARD R., KRIEG F., 1986. Mise en évidence d'un flux génétique entre populations naturelles de truites fario et souche de repeuplement dans deux rivières de Corse. Bull. Fr. Pêche Piscic., 303, 134-140.

GUYOMARD R., 1989. Diversité génétique de la truite commune. Bull. Fr. Pêche Piscic., 314, 118-135.

HAMILTON K.E., FERGUSON A., TAGGARD J.B., TOMASSON T., WALKER A., FAHY E., 1989. Post - glacial colonisation of brown trout, Salmo trutta L. : Ldh-5 as a phylogeographic marker locus. J. Fish Biol., 35, 651-664.

KARAKOUSIS Y., TRIANTAPHYLLIDIS C.D., 1990. Genetic structure and differentiation among Greek brown trout (Salmo trutta L.) populations. Heredity, 64, 297-304.

KRIEG F., 1984. Recherche d'une différenciation génétique entre populations de Salmo trutta. Thèse de 3ème cycle, Université de Paris-Sud, Orsay, 92 p.

KRIEG F., GUYOMARD R., 1985. Population genetics of French brown trout (Salmo trutta L.) : large geographical differentiation of wild populations and high similarity of domesticated stock. Genet. Sel. Evol., 17 (2), 225-242.

MORAN P., PENDAS A.M., GARCIA-VAZQUEZ E., IZQUIERO J., 1991. Failure of a stocking policy of hatchery reared brown trout, Salmo trutta L., in Asturias, Spain, detected using $\mathrm{LDH}-5^{\star}$ as a genetic marker. J. Fish Biol., 39 (suppl.A), 117-121.

PASTEUR N., PASTEUR G., BONHOMME F., CATALAN J., BRITTON-DAVIDIAN J., 1987. Manuel de génétique par électrophorèse des protéines. Lavoisier, Paris, 217 p.

SCHERRER B., 1984. Biostatistique. Ed. Gaëtan Morin, Québec, 850 p.

TAGGART J.B., FERGUSON A., 1986. Electrophoretic evaluation of a supplemental stocking programme for brown trout, Salmo trutta L. Aquacult. Fish. Mgmt, 17, 155-162.

THIBAULT M., 1983. Les transplantations de salmonidés d'eau courante en France, saumon atlantique (Salmo salar L.) et truite commune (Salmo trutta L.). C. R. Soc. Biogéogr., 59 (3c), 405-420.

VUORINEN J., 1984. Reduction of genetic variability in hatchery stock of brown trout, Salmo trutta L. J. Fish Biol., 24, 339-348. 